\title{
Citrate synthase proteins in extremophilic organisms - studies within a structure-based model
}

\author{
Bartosz Różycki ${ }^{1,}$ 国 and Marek Cieplak ${ }^{1}$ \\ Institute of Physics, Polish Academy of Sciences, \\ Al. Lotników 32/46, 02-668 Warsaw, Poland
}

(Dated: 17 October 2018)

\begin{abstract}
We study four citrate synthase homodimeric proteins within a structure-based coarse-grained model. Two of these proteins come from thermophilic bacteria, one from a cryophilic bacterium and one from a mesophilic organism; three are in the closed and two in the open conformations. Even though the proteins belong to the same fold, the model distinguishes the properties of these proteins in a way which is consistent with experiments. For instance, the thermophilic proteins are more stable thermodynamically than their mesophilic and cryophilic homologues, which we observe both in the magnitude of thermal fluctuations near the native state and in the kinetics of thermal unfolding. The level of stability correlates with the average coordination number for amino acids contacts and with the degree of structural compactness. The pattern of positional fluctuations along the sequence in the closed conformation is different than in the open conformation, including within the active site. The modes of correlated and anticorrelated movements of pairs of amino acids forming the active site are very different in the open and closed conformations. Taken together, our results show that the precise location of amino acid contacts in the native structure appears to be a critical element in explaining the similarities and differences in the thermodynamic properties, local flexibility and collective motions of the different forms of the enzyme.
\end{abstract}

Keywords: proteins, native structure, citrate synthase, thermal stability, fluctuations, coarse-grained models, structure-based models, molecular dynamics simulations

\section{INTRODUCTION}

The primary characteristics of globular proteins are their native structures and sequences of amino acids. An evolutionary divergence from a common ancestor may lead diverse sequences to fold to nearly the same native structure. One particularly interesting example is citrate synthase (CS), an enzyme that is found in most living organisms, from bacteria to man ${ }^{1}$. It acts as a part of the Krebs cycle that generates $\mathrm{ATP}^{2}$. The native structure of CS from a bacteria living in hot hydrothermal vents is superimposable with that of CS from a bacteria living under the conditions of extreme cold $\underline{\underline{1}}$ and yet properties of these proteins, such as the thermodynamic stability, are distinct so that the enzymatic action - conversion of acetyl-CoA and oxaloacetate into $\mathrm{CoA}$ and citrate $^{3}-$ is executed properly.

In this paper, we investigate to what extent structurebased coarse-grained models, or rather their one specific implementation, can capture physical differences between proteins which belong to the same fold. We study four CS proteins and demonstrate that their properties are indeed distinct in the model due to the existence of slight differences in the native structures. In particular, the model thermophilic proteins are found to have stronger thermodynamic stability than those which are cryophilic (an equivalent term is: psychrophilic). However, we also find that the root-mean-square single-site fluctuations are

\footnotetext{
a) Electronic mail: rozycki@ifpan.edu.pl
}

alike within the very region of the active site of the thermophilic and cryophilic forms, even though they are distinct in other parts of the proteins.

The structure-based dynamical models follow from Go's idea ${ }^{4} \underline{-6}$ that kinetic and thermodynamic properties of proteins should depend primarily on the geometry of the native structure and less so directly on the specificity of the sequence. There are many variants of such models (see, for instance, Refs $\underline{\underline{7}-12}$ ). They are not equivalent, but their predictions are expected to be most accurate in a vicinity of the native state - a situation encountered, for instance, during stretching manipulations ${ }^{13}-16$. It has also been argued that protein folding processes may be proceeding as in the Go-like models due to minimization of hindrances to folding $17-22$. Large-scale conformational changes in proteins can be explored by multi-state Go-type models 23,24 and mixed elastic network models 25 . In contrast, standard elastic network models, which are less demanding computationally than molecular dynamics simulations, are known to capture local fluctuations about the native state $\frac{26}{}$. Here, we employ a standard Go-type model to examine thermal stability, local flexibility, collective motions and unfolding kinetics of CS enzymes functioning in organisms that live in very different environmental conditions.

The adaptations of life to different environmental conditions can be observed at various levels of organization, including the molecular level. The composition and structure of proteins from organisms living under extreme conditions $27-29$ are known to correlate with the character of the environment. For instance, thermophilic organisms, i.e. those thriving between $45^{\circ} \mathrm{C}$ and $120^{\circ} \mathrm{C}$, tend 
to contain proteins with smaller cavities, bigger number of ionic bonds, increased polarity of exposed surfaces, an increased content of charged residues and tryptophan, and a smaller content of phenylalanine, methionine and asparagine compared to proteins in mesophilic organisms ${ }^{30}-33$. On the other hand, cryophilic organisms, i.e. those which grow and reproduce between about $-20^{\circ} \mathrm{C}$ to $+10^{\circ} \mathrm{C}$, tend to contain proteins with larger catalytic cavities, reduced content of proline and arginine (to make the backbone more flexible), increased content of clusters of glycines, less hydrophobic cores (to make the protein less compact), a higher proportion of nonpolar groups on the surface, and an increased content of negative charges on the surface (to facilitate interactions with the solvent) $34-37$. Structure-based models cannot explicitly take all of these many detailed chemical features into account, but these features lead to a particular structure of the native conformation. Therefore, such models can tell the thermophilic and cryophilic proteins apart even if they belong to the same fold. We shall inquire here - to what extent. These models also identify properties that are similar. We illustrate these aspects here by considering CS.

$\mathrm{CS}$ is an $\alpha$-protein homodimer. Fig. 11 shows an example of the native conformation - it is for CS from Pyrococcus furiosus with the Protein Data Bank (PDB) code 1AJ8 and atomic coordinates resolved for 741 amino acids ${ }^{38}$. It is formed of two identical subunits, each with its own active site. Each of the subunits comprises two domains - a small domain, which comprises five $\alpha$-helices, and and a large domain which contains $13 \alpha$-helices. In other species, the number of the helices in the large domain varies between 11 and 15 . The substrate binding site is located in a cleft between the two domains.

Two kinds of conformations of CS have been identified: one is termed 'open' and the other 'closed'. CS is in the 'open' conformation when the active site is not occupied and is thus free to bind substrate molecules for the enzyme catalysis. When the substrate binds, the small domain undergoes a rotation, sealing the substrate binding site and forming the 'closed' conformation. A number of crystal structures of CS from different organisms are available in the PDB. The five structures we have considered in our study are summarized in Table [ It would be ideal to also consider an open form of CS form a cryophilic organism, but they do not seem to be available in the PDB.

\section{METHODS}

We use a coarse-grained continuum representation for CS configurations in which only the positions of $\mathrm{C}_{\alpha}$ atoms are retained in the molecular dynamics simulations. However, the contact map, i.e. a list of nonbonded interactions is determined based on the all-atom coordinates. The configurational energy of the model

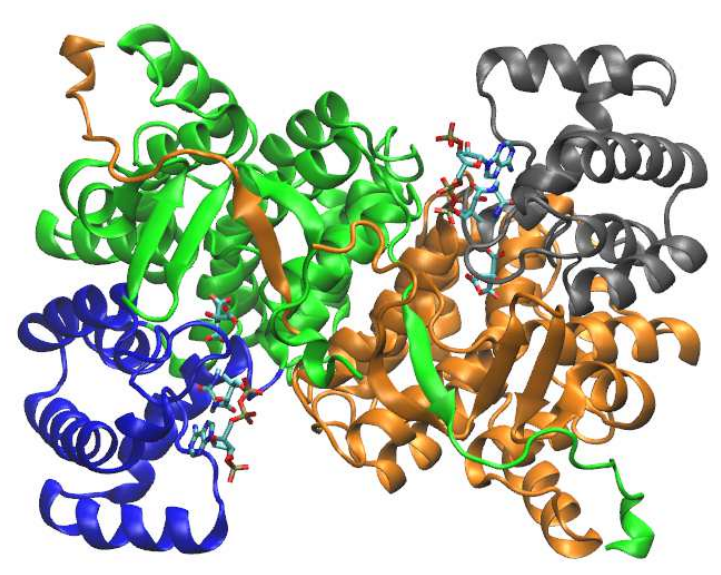

FIG. 1. Structure of citrate synthase homodimer from Pyrococcus furiosus in the closed conformation (PDB code 1AJ8). The reaction products (citrate and $\mathrm{CoA}$ ) are shown in the stick representation. The small and large domain in chain A (first monomer) are shown in blue and green, respectively, whereas those in chain B (second monomer) in gray and orange.

\begin{tabular}{|c|c|c|c|}
\hline $\begin{array}{c}\text { PDB code } \\
\text { and } \\
\text { reference }\end{array}$ & $\begin{array}{c}\text { confor- } \\
\text { mation }\end{array}$ & organism & $\begin{array}{c}\text { optimal } \\
\text { growth } \\
\text { temperature }\end{array}$ \\
\hline 1AJ8 $^{38}$ & closed & Pyrococcus furiosus & $100^{\circ} \mathrm{C}$ \\
\hline $2 \mathrm{CTS}^{39}$ & closed & Sus scrofa (pig) & $37^{\circ} \mathrm{C}$ \\
\hline $1 \mathrm{~A}^{40}{ }^{40}$ & closed & Antarctic bacterium & $0^{\circ} \mathrm{C}$ \\
\hline 2IBP $^{41}$ & open & Pyrobaculum aerophilum & $100^{\circ} \mathrm{C}$ \\
\hline 1CTS $^{39}$ & open & Sus scrofa (pig) & $37^{\circ} \mathrm{C}$ \\
\hline
\end{tabular}

TABLE I. Structures of CS from different organisms used in this study. Structures of the closed conformation contain the reaction products (CoA and citrate). The products are absent in the open conformation structures.

$$
\begin{aligned}
\operatorname{reads}^{7.8,42} & \\
E\left(X, X_{0}\right) & =\sum_{\text {bonds }} K_{r}\left(r-r_{0}\right)^{2}+\sum_{\text {angles }} K_{\theta}\left(\theta-\theta_{0}\right)^{2} \\
& +\sum_{\text {dihedral }}\left[K_{\phi}^{(1)}\left[1-\cos \left(\phi-\phi_{0}\right)\right]\right. \\
& \left.+K_{\phi}^{(3)}\left[1-\cos \left(3 \phi-3 \phi_{0}\right)\right]\right] \\
& +\sum_{\text {NC }(|i-j|>3)} \epsilon\left[5\left(\frac{r_{0 i j}}{r_{i j}}\right)^{12}-6\left(\frac{r_{0 i j}}{r_{i j}}\right)^{10}\right] \\
& +\sum_{\text {NNC }(|i-j|>3)} \epsilon\left(\frac{\rho_{0}}{r_{i j}}\right)^{12}
\end{aligned}
$$

Here, $r$ and $r_{0}$ denote the distances between two subsequent residues at configurations $X$ and $X_{0}$, respectively, 
where the reference configuration $X_{0}$ corresponds to the native state. The summation over bonds includes peptide and disulfide bonds (the latter are present in 2IBP, one in each monomer). Analogously, $\phi$ and $\phi_{0}$ represent the bond angles formed by three subsequent residues along the amino acid chain at configurations $X$ and $X_{0}$, respectively. Next, $\theta$ and $\theta_{0}$ are the dihedral angles formed by four sequential residues at configurations $X$ and $X_{0}$. The coupling parameters in the first three terms in Eq. (1D) are taken to be as in Ref.. , i.e., $K_{r}=100 \epsilon / \AA^{2}, K_{\theta}=20 \epsilon$, $K_{\phi}^{(1)}=\epsilon$, and $K_{\phi}^{(3)}=\epsilon / 2$.

The fourth term in Eq. (11) describes the native interactions. Here, $r_{i j}$ and $r_{0 i j}$ denote the distances at configurations $X$ and $X_{0}$, respectively, between residues $i$ and $j$ forming the native contacts. The native contacts are identified as in Ref $\underline{13,14,42}$, i.e., by using an overlap criterion $\underline{43}$ applied to the coordinates of all heavy atoms in the native structure. The heavy atoms are assigned enlarged van der Waals spheres and if there is a pair of atoms for which one finds spheres that overlap in the native state then the corresponding pair of amino acids is considered as forming a native contact. Physically, these contacts are due to hydrogen bonds and ionic bridges. Only contacts with $|i-j|>3$ are included in the contact map. The last term in Eq. (11) describes repulsion between non-native contacts, with $\rho_{0}=4 \AA$.

The model described by Eq. (11) is different from the one used in our recent studies of protein stretching $\underline{13}-15$ : the contact potential is of the 10-12 type, and not 6-12, and the local backbone stiffness is given by the usual bond-angle and dihedral-angle potentials instead of a term involving the local chirality (which effectively includes the dihedral term but not the bond angle terms ${ }^{11}$ ). The 6-12 model yields amino-acid positional fluctuations substantially larger than those found in experiments through the temperature factors, and coming close to the experimental data requires rescaling as in Ref. $\underline{44}$. Nevertheless, we expect that the calibration of $\epsilon$ is of the same order, i.e. $\epsilon \approx 110 \mathrm{pN} \AA$. Thus the room temperature should be in the vicinity of $0.35 \epsilon / k_{B}$, where $k_{B}$ denotes the Boltzmann constant.

In our coarse-grained simulations, the citrate and CoA molecules (present in the closed structures of CS) are represented by one and four beads, respectively. The contacts between CS and citrate, and between CS and CoA, are found using the same all-atom overlap criterion as the native contacts between the amino acids within CS. To avoid dissociation of the citrate and CoA molecules from CS during simulations, these contacts are replaced by harmonic bonds analogous to the $\mathrm{C}_{\alpha}$ - $\mathrm{C}_{\alpha}$ pseudobonds with the spring constant $K_{r}=100 \epsilon / \AA^{2}$.

To study thermodynamic properties of the CS dimers, we performed overdamped Langevin dynamics simulations using in-house software $\frac{15,42}{2}$. The simulations were carried out at 31 different temperatures $T$ distributed uniformly in the interval from $0.1 \epsilon / k_{B}$ to $0.7 \epsilon / k_{B}$. Each simulation was $10^{5} \tau$ long and it was preceded by a $10^{4} \tau$ equilibration run. The unit of time, $\tau$, is of order $1 \mathrm{~ns}$. At these temperatures and time scales the dimers never dissociate or unfold. We observe only small and moderate deviations from the native state as measured by RMSD, see Figs. 20 and $3 \mathrm{~b}$ in the next section.

In the course of the simulations, we monitor the number of contacts

$$
m(t)=\sum_{i=1}^{M} \theta\left(d_{i j}-r_{i j}(t)\right)
$$

where $M$ is the total number of contacts in the native state, $r_{i j}(t)$ is the distance between residues $i$ and $j$ at simulation time $t, d_{i j}=1.2 r_{0 i j}$ is a cutoff distance, and $\theta$ is the the Heaviside function: $\theta(x)=1$ if $x \geq 0$ and $\theta(x)=0$ if $x<0$. To assess thermal stability of the enzyme, we compute the probability of finding the enzyme in the native state

$$
P_{0}=\left\langle\delta_{m, M}\right\rangle
$$

as a function of temperature $T$, where the brackets denote time average after equilibration, and $\delta_{m, M}$ is the Kronecker delta: $\delta_{m, M}=1$ if $m=M$ and $\delta_{m, M}=0$ otherwise. Notice that the definition of $P_{0}$ involves counting conformations in which all native contacts are present. The native state probability as given by Eq. (3) is thus different from the average fraction of contacts

$$
Q=\left\langle\frac{m}{M}\right\rangle
$$

We define temperature of thermodynamical stability, $T_{f}$, as one at which $P_{0}=\frac{1}{2}$. This temperature is different and substantially lower than the temperature at which $Q=\frac{1}{2}$. Our definition of $T_{f}$ yields values of $T$ that are in a vicinity of temperatures at which smaller proteins fold optimality $\stackrel{42}{ }$.

To describe how far a particular configuration has departed from the native state, we compute the root mean square deviation

$$
\operatorname{RMSD}(t)=\left[\frac{1}{N} \sum_{i=1}^{N}\left(\vec{r}_{i}(t)-{\overrightarrow{r_{i}}}^{\mathrm{NAT}}\right)^{2}\right]
$$

where ${\overrightarrow{r_{i}}}^{\mathrm{NAT}}$ denote the positions of $\mathrm{C}_{\alpha}$ atoms in the native state and $\overrightarrow{r_{i}}$ are positions of the $\mathrm{C}_{\alpha}$ atoms at time $t$ after superimposing on the native structure. We use the Kabsch algorithm ${ }^{45}$ to superimpose the instantaneous structures on the native structure. After equilibration, RMSD fluctuates around its average value, $\langle\mathrm{RMSD}\rangle$, which is a function of temperate $T$.

To quantify the local flexibility of the enzyme, we compute the root mean square fluctuation (RMSF) of each residue

$$
\delta_{i}=\left[\left\langle{\overrightarrow{r_{i}}}^{2}\right\rangle-\left\langle\vec{r}_{i}\right\rangle^{2}\right]^{1 / 2}
$$

Here $\overrightarrow{r_{i}}$ is the position of the $i$-th $\mathrm{C}_{\alpha}$ atom, and the angle brackets denote the average after superimposing on the 
native structure. The RMSFs are directly related to the so called temperature factors 26

$$
\beta_{i}=\frac{8 \pi^{2}}{3} \delta_{i}^{2}
$$

which can be determined in biomolecular crystallography experiments and are listed in PDB files.

The correlation in the motions of residues $i$ and $j$ are given by

$$
C_{i j}=\frac{\left\langle\delta \overrightarrow{r_{i}} \cdot \delta \overrightarrow{r_{j}}\right\rangle}{\left\langle\delta \vec{r}_{i}^{2}\right\rangle^{1 / 2}\left\langle\delta \vec{r}_{j}^{2}\right\rangle^{1 / 2}}
$$

where $\delta \overrightarrow{r_{i}}=\overrightarrow{r_{i}}-\left\langle\overrightarrow{r_{i}}\right\rangle$ is the displacement from the average position computed after superimposing on the native structure. Significant positive correlations mean that the residues tend to move together, possibly as part of a larger structural motif. Negative correlations imply that the two residues tend to move in opposite directions.

To study thermal unfolding and dissociation of the CS dimers, we performed overdamped Langevin dynamics simulations at elevated temperatures $T$ between $1.1 \epsilon / k_{B}$ and $1.7 \epsilon / k_{B}$. At each of temperatures we run 200 trajectories to study kinetics of the unfolding and dissociation processes.

\section{RESULTS}

\section{A. Thermodynamic properties of CS dimers}
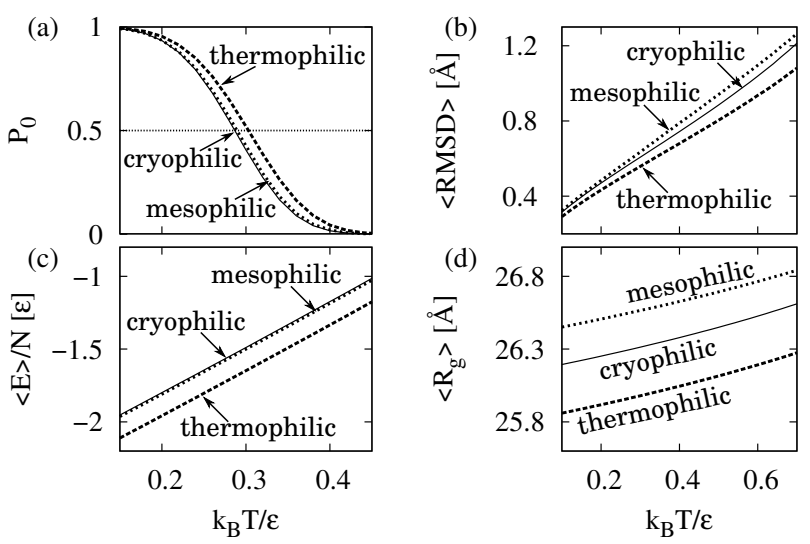

FIG. 2. Thermodynamic properties of citrate synthase from thermophilic (1AJ8, dashed lines), mesophilic (2CTS, dotted lines) and cryophilic (1A59, thin solid lines) organisms in the closed conformation. (a) The probability of finding the enzyme in the native state, $P_{0}$, (b) the average root mean square deviation (RMSD) from the native state, (c) the average internal energy per residue, and (d) the average radius of gyration, $\left\langle R_{g}\right\rangle$, as functions of temperature $T$.

We first compare some thermodynamic properties of the CS dimers from thermophilic and cryophilic organisms in the closed conformation (PDB codes 1AJ8 and
1A59, respectively). Fig. 2 shows that, at any a given $T$, the probability $P_{0}$ of finding the enzyme in the native state is larger for the thermophilic CS than for the cryophilic one. In particular, $T_{f}$ is $0.303 \epsilon / k_{B}$ and $0.287 \epsilon / k_{B}$ for the thermophilic and cryophilic CS dimers, respectively. With our calibration of $\epsilon$, the difference is of order $14^{\circ} \mathrm{C}$.

Fig. 20 shows that, at any given $T,\langle$ RMSD $\rangle$ is larger for the cryophilic CS than for the thermophilic one. Thus the cryophilic CS undergoes larger thermal fluctuations than the thermophilic CS at the same $T$. The internal energy per residue, shown in Fig 2 r, is lower by about $0.2 \epsilon$ for the thermophilic CS compared to the cryophilic CS, independent of the value of $T$. This result is consistent with the data in Fig 2 a showing that the thermophilic CS exhibits better thermal stability than the thermophilic CS.

Interestingly, the thermophilic CS appears slightly more compact than the cryophilic CS, as evidenced by the time-averaged radius of gyration, $\left\langle R_{g}\right\rangle$, plotted against $T$, see Fig. 2] (note that these two dimers comprise comparable numbers of residues, 740 and 754 , respectively). The difference in $\left\langle R_{g}\right\rangle$ is about $0.4 \AA$ or about $1.5 \%$ of the native $R_{g}$. We have checked that, at a given temperature $T$ in the range between $0.1 \epsilon / k_{B}$ and $0.7 \epsilon / k_{B}$, the standard deviation of the instantaneous $R_{g}$ values is smaller than $0.1 \AA$, both for the cryophilic and thermophilic forms of CS. Thus the observed difference in the values of $\left\langle R_{g}\right\rangle$ is small but statistically relevant.

We next discuss the thermodynamic properties of the mesophilic CS in the closed conformation (with the PDB code 2 CTS). We find that, at any given temperature, $P_{0}$ for the mesophilic CS is smaller than for the thermophilic one and only slightly bigger than for the cryophilic CS, see Fig. 2 2 . Its stability temperature is $T_{f}=0.289 \epsilon / k_{B}$. The stability difference, as measured by alterations in $T_{f}$, between the thermophilic and mesophilic CS dimers in the closed conformation is of order of $12^{\circ} \mathrm{C}$.

Interestingly, out of the three CS dimers in the closed conformation, the mesophilic one shows the fastest increase in $\langle$ RMSD $\rangle$ with $T$, see Fig. 2 b. At any given $T$, the internal energy per residue for the mesophilic and cryophilic CS dimers in the closed conformation are almost the same, see Fig. 2r. This observation is consistent with the results shown in Fig 2 a, namely, that these two forms are characterized by very similar $P_{0}(T)$ curves. The mesophilic CS dimer comprises 874 amino acid residues whereas its cryophilic and thermophilic homologues - only 754 and 740 residues, respectively. This explains why the mesophilic form has the larges radius of gyration, as shown in Fig. 2 $\mathrm{d}$.

We next compare the thermodynamic properties of CS dimers from thermophilic and mesophilic organisms in the open conformation (PDB codes 2IBP and 1CTS, respectively). As shown in Fig. 3a, the thermophilic CS exhibits larger $P_{0}(T)$ and is thus thermodynamically more stable than the mesophilic CS. The values of $T_{f}$ are $0.285 \epsilon / k_{B}$ and $0.274 \epsilon / k_{B}$ for the thermophilic and 

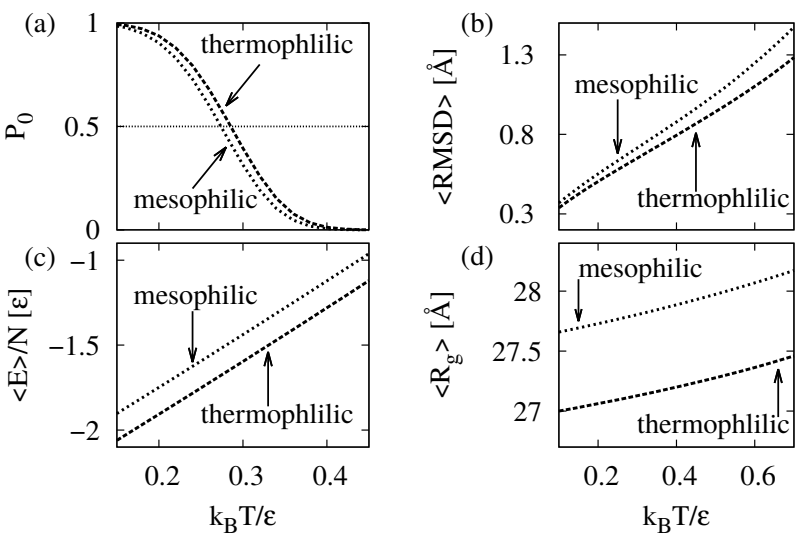

FIG. 3. Similar to Fig. 2 but for citrate synthase from thermophilic (2IBP) and mesophilic (1CTS) organisms in the open conformation.

mesophilic CS, respectively. The difference is of order $10^{\circ}$ C. Fig. $3 \mathrm{~b}$ shows that the mesophilic CS exhibits larger $\langle\operatorname{RMSD}\rangle(T)$ and is thus more susceptible for thermal fluctuations than the thermophilic CS. A larger thermal stability of the thermophilic CS can be also inferred from Fig. 3r which shows the internal energy per residue as a function of $T$. We note also that the thermophilic CS is more compact than than the mesophilic CS, see Fig. 3 $\mathrm{d}$. Overall, we observe that the qualitative differences between the proteins belonging to the organisms preferring high and low temperatures do not depend on whether the structures are closed or open.

It is instructive to compare the open and closed CSs from the same organism (with PDB codes 1CTS and $2 \mathrm{CTS}$, respectively). We note that the closed form is more compact than the open one. Its radius of gyration is smaller by $1.2 \AA$. The closed form exhibits a slower decrease of $P_{0}$ with $T$. As mentioned before, $T_{f}$ is $0.289 \epsilon / k_{B}$ for the closed form and $0.274 \epsilon / k_{B}$ for the open one. Since the two forms have identical sequences, the difference in $T_{f}$ should be related to the number of native contacts, which is 2060 in the open conformation and 2138 in the closed conformation.

The higher thermodynamic stability of the thermophilic proteins is expected and required by the function. It is interesting to ask, however, what features of the native structure encode this information. It has been shown recently, in a similar model, that effective stiffness of virus capsids, as measured in nanoindentation experiments, correlates with average coordination number, $z \underline{46}$. Specifically, the corresponding Young modulus is proportional to $(z-6)^{2}$. The average coordination number is defined as

$$
z=2 \frac{\# \text { native contacts }+ \text { \#bonds }}{\text { \#residues }}
$$

and it describes how many neighbors, on an average, any residue has. It is determined in the native state and the notion of neighborhood relates to the dynamics of the system. Table $\amalg$ demonstrates that the values of $T_{f}$ correlate with $z$ both in the open and closed conformation. The coordination number can be determined both for the dimers and individual monomers. The corresponding quantities $z_{\text {dimer }}$ and $z_{\text {monomer }}$ differ because there are contacts formed at the interface of the two monomers. The number of the interfacial contacts in 1AJ8 is 289; in 2 CTS it is 302 , whereas in $1 \mathrm{~A} 59$ it is only 266 . For the closed forms the numbers are 322 for 2IBP and 252 for 1 CTS. Whichever quantity one takes, $z_{\text {dimer }}$ or $z_{\text {monomer }}$, the correlation with $T_{f}$ is evident, which indicates that both bulk and interfacial contacts contribute to the larger stability of the thermophilic forms. The larger value of $z$ is also consistent with the tighter packing of residues in thermophilic enzymes.

It is interesting to ask whether the stability temperature is correlated with the average contact order

$$
C O=\frac{1}{M N} \sum_{i j} \Delta_{i j}
$$

Here, $N$ is the number of residues in an amino acid chain, where $M$ is the total number of the native contacts, and $\Delta_{i j}=|i-j|$ is the sequence separation between residues $i$ and $j$ that form a native contact. Note that the contact order is uniquely defined only for single amino acid chains (monomers). The values of the average contact order as calculated from the contact maps of the five CS forms are given in the last column of Table Interestingly, we find no correlation between $C O$ and $T_{f}$. A similar conclusion has been reached in Ref. $\underline{\underline{47}}$.

\section{B. The temperature factors and RMSF}

To validate our simulations, we compare experimental and simulational temperature factors of the different CSs, see Fig. 4. The simulations have been done at $T=0.3 \epsilon / k_{B}$ which is close to $T_{f}$. Although the temperature factors in a crystal may in general differ from those in a solution, this approach has been widely used to parametrize elastic network models 26 .

We obtain very good agreement for the 2IBP and 1A59 structures, see panels (a) and (b) in Fig. 4. For 1AJ8, see Fig. 4(c), the simulation pattern closely resembles the distribution of the crystallographic $\beta$ factors but its magnitude is too small. However, simulations at $T=$ $0.4 \epsilon / k_{B}$ fit the experiment much better, see the blue line in Fig 4(c). We do not compare results for 1CTS because the resolution of the mesophilic CS structure in the open conformation (1CTS) is only $2.7 \AA$. The resolution for the structures of 2IBP, 1A59, and 1AJ8 is much better and is equal to $1.6 \AA, 2.1 \AA$, and $1.9 \AA$ respectively.

We note that the average crystallographic $\beta$ factor, $\bar{\beta}=\frac{1}{N} \sum_{i=1}^{N} \beta_{i}$, is $\bar{\beta}=21 \AA^{2}$ for the thermophilic CS in the closed conformation (1AJ8), and $\bar{\beta}=14 \AA^{2}$ for the cryophilic CS in the closed conformation (1A59). Importantly, in both cases the protein crystals have been re- 


\begin{tabular}{|c|c|c|c|c|c|c|}
\hline organism type & conformation & PDB code & $k_{B} T_{f} / \epsilon$ & $z_{\text {dimer }}$ & $z_{\text {monomer }}$ & $C O$ \\
\hline \hline thermophilic & closed & 1AJ8 & 0.303 & 7.31 & 6.54 & 0.0859 \\
\hline mesophilic & closed & 2CTS & 0.289 & 7.01 & 6.33 & 0.0784 \\
\hline cryophilic & closed & 1 A59 & 0.287 & 6.99 & 6.29 & 0.0876 \\
\hline \hline thermophilic & open & 2IBP & 0.285 & 7.04 & 6.25 & 0.0846 \\
\hline mesophilic & open & 1CTS & 0.274 & 6.71 & 6.14 & 0.0811 \\
\hline
\end{tabular}

TABLE II. In both the open and closed conformation, $T_{f}$ correlates with the average coordination number both for CS dimers $\left(z_{\text {dimer }}\right)$ and monomers $\left(z_{\text {monomer }}\right)$.
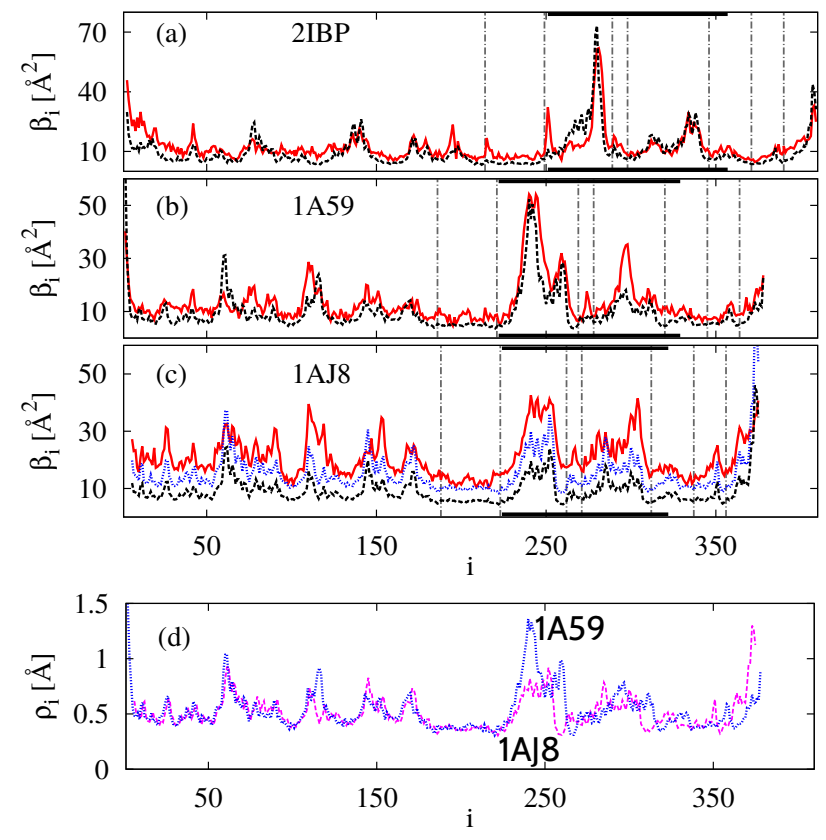

FIG. 4. Temperature factors $\beta_{i}$ versus the residue number $i$ from X-ray crystallography experiments (see the solid lines in red) and results of simulations at $T=0.3 \epsilon / k_{B}$ (see the dashed lines in black). The PDB codes of the different enzymes are given in figure panels. The vertical lines indicate the residues that form the active site as listed in Table S1 in Supplementary Material ${ }^{53}$. The thick horizontal lines show the location of the small domain. The blue dashed line in panel (c) shows the result of simulations at $T=0.4 \epsilon / k_{B}$. Panel (d) shows RMSF along the sequence, $\delta_{i}$, computed at $T=T_{f}$ for $1 \mathrm{AJ} 8\left(T_{f}=0.303 \epsilon / k_{B}\right.$; see the dashed line in magenta) and 1 A59 $\left(T_{f}=0.287 \epsilon / k_{B}\right.$; see the dotted line in blue).

ported to be obtained at the room temperature. The experimental data would thus imply that the thermophilic $\mathrm{CS}$ is, on average, more flexible than its cryophilic homologue at the same temperature. One would certainly expect the opposite relation. This inconsistency might be a reason for the quantitative disagreement between simulations and experiment in the case of 1AJ8, see Fig. 4(c).

In all of the analyzed structures, the residues that have the largest $\beta$ factors are localized in the small domain (the region highlighted in Fig. 4), primarily in the segments that bind the CoA molecule. In contrast, the residues that bind citrate (indicated by the vertical lines in Fig. (4) have relatively small temperature factors.

When one compares the $\beta$ factors of the thermophilic and cryophilic proteins in the closed conformation, see panels (b) and (c) of Fig. 4, then they are seen to map out very similar looking patterns no matter whether one uses the experimental results at the room temperature (red solid lines in Fig. 4) or the simulational results at $T=0.3 \epsilon / k_{B}$ (black dashed lines in Fig. 4). When $\delta_{i}$ or $\beta_{i}$ are calculated at a higher $T$ for the thermophilic form and at a lower $T$ for the cryophilic form then the match in the patterns is better quantitatively. Fig. 4(d) compares $\delta_{i}$ for 1 AJ8 and 1 A59 at their respective stability temperatures. The amplitudes of the fluctuations are seen to be comparable.

\section{Fluctuations of the active site}

The citrate binding site comprises seven evolutionarily conserved amino acids: three histidine residues, three arginine residues and one aspartic acid $36,48,49$. They are depicted in Fig. 5] and summarized in Table S1 which is provided in Supplementary Material ${ }^{53}$. Three of them, which we denote here as His 2 , His 3 and $\mathrm{Asp}_{1}$, are directly involved in the chemical reaction that results in citrate formation ${ }^{49}$. They are highlighted in Table $\mathrm{S}^{53}$ in bold. $\mathrm{His}_{3}, \mathrm{Arg}_{1}$ and $\mathrm{Aps}_{1}$ reside in the small domain. His ${ }_{1}$, $\mathrm{His}_{2}, \mathrm{Arg}_{2}$ and $\mathrm{Arg}_{3}$ are located in the large domain (His is at the N-terminal end of the large domain). $\operatorname{Arg}_{3}$ is the only citrate-binding residue that resides on the other monomer.

In Fig. 4 the citrate binding residues are labeled with vertical lines. All of them exhibit relatively small spatial fluctuations, as indicated by low $\beta$ factors. Fig. 6] shows the RMSFs of these seven amino acid residues as a function of $T$. In the closed conformation, the active site in the thermophilic and cryophilic enzymes fluctuate in the same manner although, for any fixed $T$, the magnitude of fluctuations is somewhat larger in the cryophilic CS, see panels c and d in Fig. 6. The residues that exhibit largest RMSFs are $\operatorname{Arg}_{1}, \operatorname{Arg}_{3}$ and $\mathrm{His}_{1}$. The residues that fluctuate most weakly are $\mathrm{His}_{2}, \mathrm{Asp}_{1}$ and His 3 , which happen to be the three amino acids that are directly involved in the chemical reaction of citrate formation. 


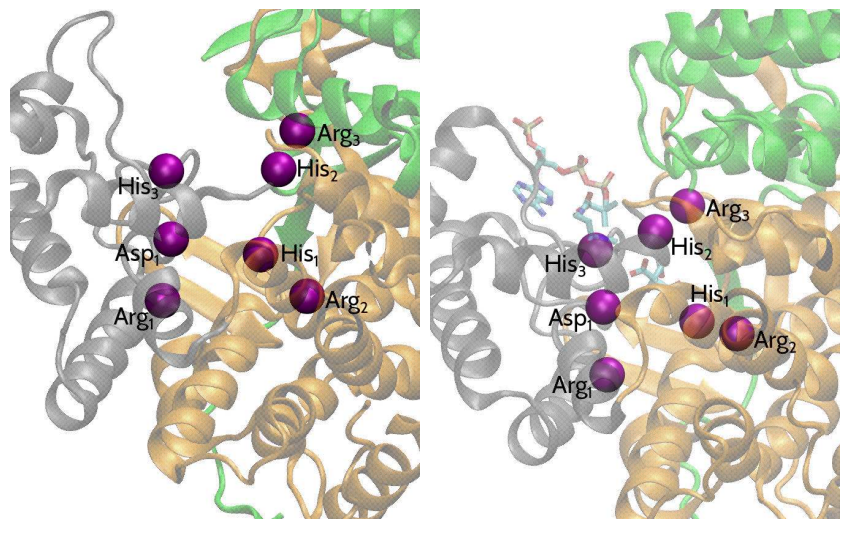

FIG. 5. Structures of the citrate binding site in the open (2IBP, left) and closed (1AJ8, right) conformations. The seven evolutionarily conserved residues that are involved in binding the citrate molecule are shown as purple spheres. The citrate and CoA molecules are shown in the stick representation. The color code in the same as in Fig. 1.

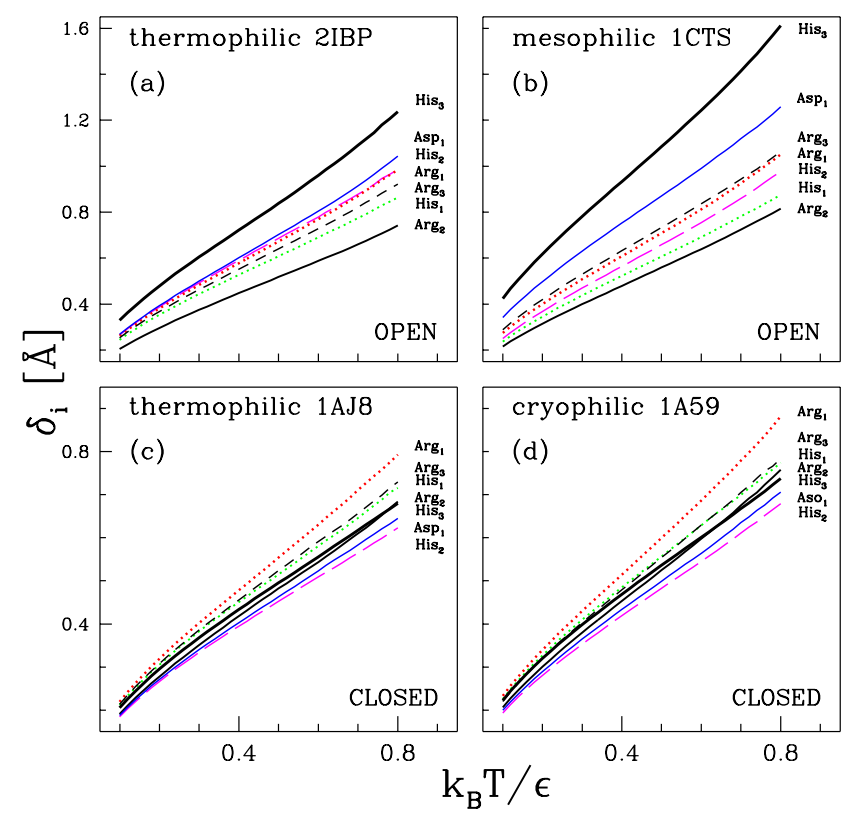

FIG. 6. RMSF $\delta_{i}$ of the seven residues forming the active site as a function of $T$.

Our results at temperatures between 0.35 and $0.45 \epsilon / k_{B}$ agree qualitatively with the results of extensive all-atom molecular dynamics simulations ${ }^{36}$, which show that at $T=300 \mathrm{~K}$ the RMSFs of the active site residues in contact with the citrate molecule are in the range between 0.4 and $0.6 \AA$, depending on the organism (thermophilic, mesophilic or cryophilic) and boundary conditions (periodic or spherical).

In the open conformation, the active site in the thermophilic and mesophilic enzymes fluctuate in a similar manner but quite differently than in the closed conformation, see panels a and b in Fig 6. Here, the residues that fluctuate the most are $\mathrm{His}_{3}$ and $\mathrm{Asp}_{1}$, which are involved in the reaction of citrate formation. The residues that exhibit smallest fluctuations are $\mathrm{Arg}_{2}$ and His $\mathrm{H}_{1}$. Also the magnitude of RMSFs is larger than in the closed conformation.

Interestingly, the three residues involved directly in the catalytic activity, $\mathrm{His}_{3}, \mathrm{His}_{2}$ and $\mathrm{Asp}_{1}$, exhibit rather small thermal fluctuations in the closed conformation, whereas in the open conformation the thermal fluctuations of these three residues are significantly enhanced. This result seems consistent with the division of functions between residues in the active site: some amino acids are tailored to binding the substrate, whereas others participate in the catalytic process.

\section{Collective motions of the active site}
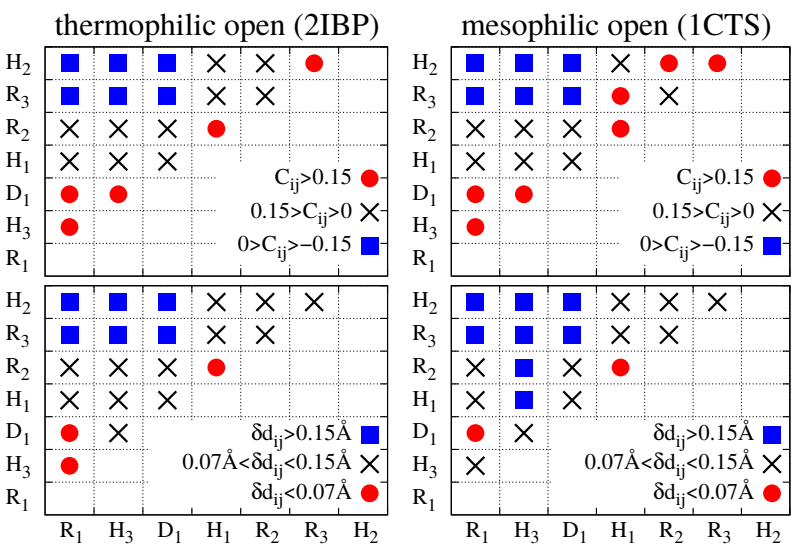

FIG. 7. Equal-time correlation coefficients (upper panels) and variation of distances (lower panels) between the seven residues forming the active site in the open conformation. The results were obtained in simulations of the thermophilic (left panels) and mesophilic (right panels) CS dimers at $T=0.3 \epsilon / k_{B}$. The largest positive correlations and the smallest distance variations are shown as red circles. The negative correlations and the largest distance variations are shown as blue squares. The intermediate cases are indicated by black crosses.

In order to identify collective motions of the active site residues, we compute the equal-time correlation coefficients $C_{i j}$ as defined in Eq. (8) for all pairs $(i, j)$ of the residues forming the active site. The upper panels of Fig. 7 show correlation levels of these pairs in the thermophilic (left panel) and mesophilic (right panel) enzymes in the open conformation. The positive correlations with $C_{i j}>0.15$ are labeled with red circles and indicate that the pairs of residues often move together in the same direction. The negative correlations are depicted as blue squares and indicate that the pairs have a tendency to move simultaneously in opposite directions. 
The results shown in Fig. 7 were obtained in simulations at temperature $T=0.3 \epsilon / k_{B}$ but we checked that the same correlation pattern persists at temperatures between $0.2 \epsilon / k_{B}$ and $0.5 \epsilon / k_{B}$.

Interestingly, all the active site residues that are located in the small domain, $\mathrm{His}_{3}, \mathrm{Arg}_{1}$ and $\mathrm{Asp}_{1}$, appear to move together as a unit, which is indicated by significant positive correlations for all the three residue pairs. The three residues in the small domain exhibit motions that are anti-correlated with the motions of $\mathrm{His}_{2}$ and $\mathrm{Arg}_{3}$, which are placed in the large domain. Therefore, the collective motions of the active site in the open conformation seem to be due to the displacements of the small domain relative to the large domain.

To further quantify this observation we compute the variations of the inter-residue distances, $\delta d_{i j}=\left\langle\left(d_{i j}-\right.\right.$ $\left.\left.\left\langle d_{i j}\right\rangle\right)^{2}\right\rangle^{1 / 2}$, where $\left\langle d_{i j}\right\rangle$ is the average distance between residues $i$ and $j$ forming the active site. The lower panels of Fig. (7illustrate the distance variations in the open conformation of the thermophilic (left panel) and mesophilic (right panel) enzymes. The smallest distance variations (red circles) are observed for the residue pairs that exhibit correlated motions with $C_{i j}>0.15$. The largest distance variations (blue squares) are observed almost exclusively for the residue pairs that exhibit negative spatial correlations and, thus, have the propensity to move in opposite directions. Importantly, these results show that the motions of largest amplitudes occur primarily between the residues located in different domains, and the smallamplitude motions are mainly between the residues residing in the same domains.

In the open conformation of the thermophilic CS, we can distinguish three groups of spatially-correlated residues: the first group comprises the three residues in the small domain, $\operatorname{Arg}_{1}, \mathrm{His}_{3}$ and $\mathrm{Asp}_{1}$; the second group consists of $\mathrm{His}_{1}$ and $\mathrm{Arg}_{2}$; and the third group is $\mathrm{His}_{2}$ and $\mathrm{Arg}_{3}$. Within each of the groups, the individual residues show a propensity to move jointly with other group members. For example, $\mathrm{His}_{1}$ and $\mathrm{Arg}_{2}$ often move together in the same direction $\left(C_{i j}=0.26\right)$ but almost independently from other residues of the active site. Interestingly, all the residues in the first group are spatially anti-correlated with the residues in the third group. These two groups can be thus visualized as the lower and upper 'jaws' moving in an up-and-down manner.

In the open conformation of the mesophilic CS, we observe more correlations between the active site residues present in the large domain. Here, the 'upper jaw' (His 2 and $\mathrm{Arg}_{3}$ ) appears to be connected more stiffly to the 'hinge' (His 1 and $\mathrm{Arg}_{2}$ ) but performs even larger motions $\left(\delta d_{i j}>0.2 \AA\right)$ against the 'lower jaw' $\left(\operatorname{Arg}_{1}, \mathrm{His}_{3}\right.$ and $\left.\mathrm{Asp}_{1}\right)$.

In the closed conformation, both in the thermophilic and cryophilic enzymes, we observe only positive correlations, $C_{i j}>0$, and rather small inter-residue distance variations, $\delta d_{i j}<0.07 \AA$, see Fig. S1 in Supplementary Material ${ }^{53}$. Here, due to the presence of the citrate and CoA molecules, the small domain is tightly bound to the large domain, and the active site residues have no freedom to perform any large-amplitude motions. Therefore, the residues in the small domain are positively correlated with those in the large domain. Interestingly, the active sites in the thermophilic and cryophilic CS exhibit the same pattern of correlations, see the top panels in Fig. S1.

\section{E. Kinetics of thermal unfolding and dissociation}

Another way to assess thermal stability of proteins is to simulate their unfolding at elevated temperatures as analyzed theoretically in Ref $\underline{50}$. The unfolding simulations start at the native state and finish when all nonlocal contacts get broken, which defines the unfolding time $t_{\text {unf }}$. Specifically, the nonlocality refers to the sequential distance $|i-j|>l$. We take $l=10$ in this study. In Ref $\underline{50}, l$ has been taken to be 4 to eliminate the local contacts in $\alpha$-helices, but this choice is too demanding computationally in the current context.

We performed unfolding simulations at high temperatures only for the CS dimers in the open conformation because dissociation of the citrate and CoA molecules from CS occurs much faster than protein unfolding or dimer dissociation (kinetic constants for the successive steps of the CS reaction at physiological conditions are detailed, for example, in Ref $\stackrel{51}{5})$. At any given $T$ between $1.1 \epsilon / k_{B}$ and $1.7 \epsilon / k_{B}$, we run 200 trajectories. We checked that $t_{\mathrm{unf}}<25000 \tau$ in all the simulation trajectories.
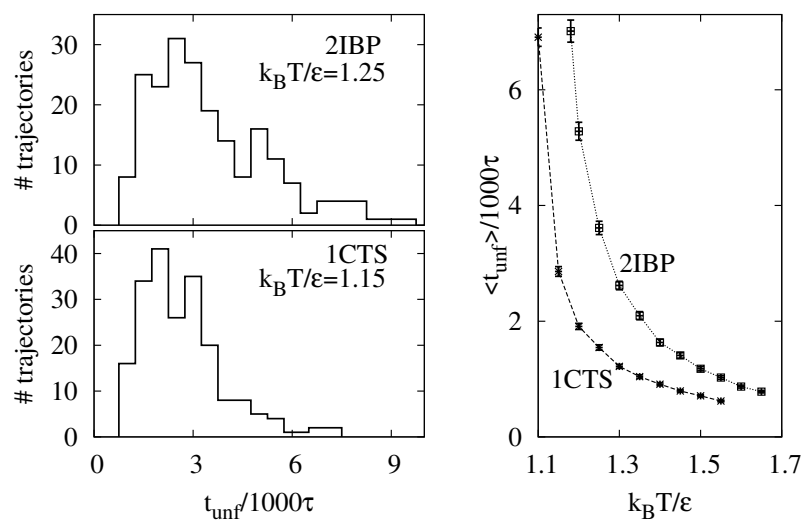

FIG. 8. Thermal unfolding of the thermophilic (2IBP) and mesophilic (1CTS) CS in the open conformation. The panels on the left hand side show histograms of the unfolding time, $t_{\text {unf }}$, at temperatures $T=1.25 \epsilon / k_{B}$ (2IBP, upper-left panel) and $T=1.15 \epsilon / k_{B}$ (1CTS, lower-left panel). The panel on the right hand side shows the average unfolding time as a function of temperature.

The histograms of $t_{\mathrm{unf}}$ for the thermophilic (2IBP) and mesophilic (1CTS) CS in the open conformation are shown in the left-hand-side panels of Fig. 8. These histograms demonstrate that the thermophilic CS unfolds slower at $T=1.25 \epsilon / k_{B}$ than the mesophilic CS at $T=1.15 \epsilon / k_{B}$, which means that the thermophilic en- 
zyme is more resistant to thermal denaturation. Also, the histograms seem to display multi-peak profiles, which suggests that there might be more than one characteristic time scale governing the thermal unfolding of the CS dimers. To further characterize the unfolding kinetics, it is instructive to calculate the fraction of the number of trajectories, $\phi_{f}(t)$, in which the unfolding event has not occurred within time $t$ from the beginning of the simulation. The fraction $\phi_{f}(t)$ provides an estimate for the probability of not unfolding CS within time $t$. As can be seen in Fig. S2 ${ }^{53}$, there is an initial lag phase in which $\phi_{f}(t)=1$. The lag phase is followed by a fast decrease of $\phi_{f}(t)$. Interestinlgy, we find that the decay of $\phi_{f}$ with $t$ is not exponential.

The right-hand-side panel of Fig. 8 shows the average unfolding time, $\left\langle t_{\text {unf }}\right\rangle$, as a function of $T$. The average is taken over 200 trajectories. The error bars correspond to the standard error of the mean. At any specified temperature, the thermophilic CS (2IBP) exhibits significantly larger unfolding time than the mesophilic CS (1CTS). This result shows that the thermophilic enzyme is thermally more stable than its mesophilic homologue. Based on the dependence of $\left\langle t_{\text {unf }}\right\rangle$ on $T$ as shown in Fig. 8, the difference in stability temperatures is of the order $0.1 \epsilon / k_{B}$, which corresponds to about $80^{\circ} \mathrm{C}$.

We use an analogous method to quantify thermal dissociation of CS dimers. The dynamics of the system is exactly the same as in the unfolding simulations but only the contacts between the monomers are monitored. The simulations start at the native state and finish when all contacts between the monomers get broken, which defines the unbinding time $t_{\mathrm{unb}}$. In the range of temperatures studied, $t_{\text {unb }}<25000 \tau$ in all the trajectories.
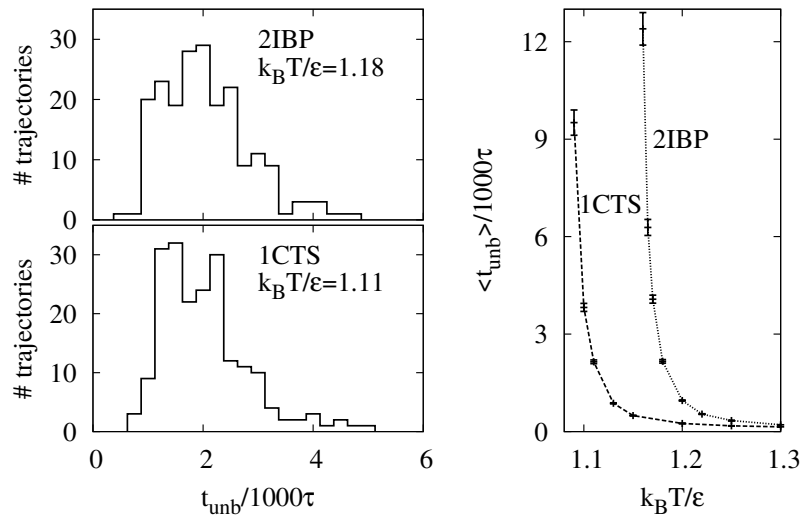

FIG. 9. Thermal dissociation of the thermophilic (2IBP) and mesophilic (1CTS) CS dimers in the open conformation. The left-hand-side panels show histograms of the unbinding times at $T=1.11 \epsilon / k_{B}(2 \mathrm{IBP})$ and $T=1.18 \epsilon / k_{B}$ (1CTS). The right-hand-side panel shows $\left\langle t_{\mathrm{unf}}\right\rangle$ as a function of $T$.

The left panels of Fig. 9 show histograms of the unbinding time for the thermophilic (2IBP) and mesophilic (1CTS) CS in the open conformation at $T=1.11 \epsilon / k_{B}$ and $T=1.18 \epsilon / k_{B}$, respectively. These data show that the thermophilic dimer is more resistant to thermal dissociation. To characterize the dissociation kinetics, we compute the fraction of the number of trajectories, $\phi_{b}(t)$, in which the unbinding event has not occurred within time $t$. Fig. S2 ${ }^{53}$ shows $\phi_{b}(t)$ for 2IBP and 1CTS at different temperatures. We note that the decay of $\phi_{b}$ with $t$ is not exponential.

The right panel of Fig. 9 shows the average unbinding time, $\left\langle t_{\mathrm{unb}}\right\rangle$, as a function of $T$. The average is taken over 200 trajectories, and the error bars correspond to the standard error of the mean. At any given temperature, $\left\langle t_{\mathrm{unb}}\right\rangle$ of the thermophilic CS dimer (2IBP) is significantly larger than that of the mesophilic enzyme (1CTS). Interestingly, the two plots of $\left\langle t_{\text {unb }}\right\rangle$ versus $T$ overlap if the temperature is shifted by about $0.07 \epsilon / k_{B}$. This temperature shift corresponds roughly to $60^{\circ} \mathrm{C}$, which is consistent with the difference in the optimal growth temperatures, see Table 1

\section{SUMMARY}

We performed Langevin dynamics simulations of CS dimers from thermophilic, mesophilic and cryophilic organisms using a coarse-grained structure-based model that does not differentiate between the ionic, hydrophobic or van der Waals interactions. In fact, the interactions between different amino acid residues are described in this model by the same potential energy function. Although this model might seem oversimplified, it correctly predicts that the thermophilic CS is thermally more stable than the mesophilic and cryophilic ones. It also yields root-mean-square fluctuates of single amino acid residues that are fully consistent with crystallographic temperature factors. Therefore, the precise location of the amino acid contacts appears to be a key element in explaining thermodynamic properties and local flexibility of enzymes.

The difference of the thermodynamical stability temperatures, $\Delta T_{f}$, can be obtained from the native state probabilities (see section IIIA). For the CS dimers from thermophilic and cryophilic organisms in the closed conformation we obtain $\Delta T_{f} \approx 14^{\circ} \mathrm{C}$; for the thermophilic and mesophilic CS dimers in the open conformation we get $\Delta T_{f} \approx 10^{\circ} \mathrm{C}$. These values are smaller than expected. The expected difference in melting temperatures between the mesophilic and thermophilic CS dimers is about $20^{\circ} \mathrm{C}$ as reported in a recent study $\stackrel{52}{\underline{2}}$. The likely reason for the fact that our model underestimates the transition temperature differences is that it lacks some relevant sequence effects. As discussed in section [I], the atomic structures of the CS proteins are used in the Gotype model only to construct the contact maps whereas the chemical composition of the proteins is not directly included in the energy function of the model as given by Eq. (1).

The temperature of optimal folding, $T_{f}$, is one measure of the thermodynamic stability of proteins. Another 
measure is provided by the kinetics of thermal unfolding (see section IIIE). These two measures are quite different as they refer to equilibrium properties near the native state and dynamic properties away from the native state, respectively. One measures the frequency of the situations in which all contacts are present simultaneously, and the other focuses on rupture events up to a given sequential length. The stability temperature difference obtained from the thermal unfolding of CS dimers from thermophilic and mesophilic organisms (see Fig. 8) is of the order of $80^{\circ} \mathrm{C}$. We note that this value can be affected by the partition of the native contacts into local and nonlocal (we used the sequential distance cut-off $l=10$ in our analysis). On the other hand, the stability temperature difference obtained from the thermal dissociation of CS dimers from thermophilic and mesophilic organisms is about $60^{\circ} \mathrm{C}$ (see Fig. 9). We note that this value is unaffected by the choice of the sequential distance cutoff, $l$, as we take into account all inter-monomer contacts in the analysis of the simulation data. Interestingly, this temperature difference, $60^{\circ} \mathrm{C}$, is consistent with the difference in the optimal growth temperatures as given in Table I.

We analyzed thermal fluctuates and collective motions of the active site in CS enzymes from different organisms, both in the open and closed conformations. We find that the three residues that are directly involved in the chemical reaction of citrate formation exhibit rather small fluctuations in the closed conformation. In the open conformation, however, the thermal fluctuations of these three residues are significantly enhanced. We also find that the collective motions of the active site in the open conformation are mainly due to the movements of the small domain relative to the large domain. In the open conformation, due to the presence of the citrate and CoA molecules, the small domain is tightly bound to the large domain, and the active site residues have no freedom to perform any large-amplitude collective motions. Taken together, our results show that the relatively simple structure-based model correctly captures the similarities and differences in thermodynamic and kinetic properties of the different forms of the CS enzyme.

\section{Acknowledgments}

This work has been supported by the Polish National Science Center grant No. 2012/05/B/NZ1/00631 (BR) and by the ERA-NET grant FiberFuel (MC).

\footnotetext{
${ }^{1}$ Nick Lane, Life ascending: The ten great inventions of evolution. W.W. Norton \& Co., New York (2009), ISBN 0-393-06596-0.

${ }^{2}$ H. A. Krebs and P. D. J. Weitzman, Krebs' citric acid Cycle: half a century and still turning. Biochemical Society, London (1987), ISBN 0-904498-22-0.

${ }^{3}$ D. L. Nelson and M. M. Cox, Lehninger Principles of Biochemistry. Fourth Edition. W.H. Freeman \& Co. (2005).

${ }^{4}$ Y. Ueda, H. Taketomi, and N. Go, Studies on protein folding, unfolding and fluctuations by computer simulation. A threedimensional lattice model of lysozyme, Biopolymers 17, 15311548 (1978).
}

${ }^{5} \mathrm{H}$. Abe and N. Go, Noninteracting local-structure model of fold- ing and unfolding transition in globular proteins. II. Application to two-dimensional lattice proteins, Biopolymers 20, 1013-1031 (1981).

${ }^{6} \mathrm{~N}$. Go, Theoretical studies of protein folding, Annu. Rev. Biophys. Bioeng. 12, 183-210 (1983).

${ }^{7}$ T. X. Hoang and M. Cieplak, Molecular dynamics of folding of secondary structures in Go-like models of proteins, J. Chem. Phys. 112, 6851-6862 (2000).

${ }^{8}$ C. Clementi, H. Nymeyer, and J. N. Onuchic, Topological and energetic factors: what determines the structural details of the transition state ensemble and en-route intermediates for protein folding? An investigation for small globular proteins, J. Mol. Biol. 298, 937-953 (2000).

${ }^{9} \mathrm{~J}$. Karanicolas and Ch. L. Brooks III, The origins of the asymmetry in the folding transition states of protein $L$ and $G$, Prot. Sci. 11, 2351-2361 (2002).

${ }^{10}$ Y. Levy, P.G. Wolynes, and J. Onuchic, Protein topology determines binding mechanism, Proc. Natl. Acad. Sci. USA, 101, 511516 (2004).

${ }^{11}$ J. I. Sułkowska and M. Cieplak, Selection of optimal variants of Go-like models of proteins through studies of stretching, Biophys. J. 95, 3174-3191 (2008).

${ }^{12}$ J. K. Noel and J. N. Onuchic, The Many Faces of Structure-Based Potentials: From Protein Folding Landscapes to Structural Characterization of Complex Biomolecules, in Computational modeling of biological systems: From molecules to pathways. ed. N. Dokholyan, Springer Science+Business Media, LLC (2012).

${ }^{13}$ J. I. Sułkowska and M. Cieplak, Mechanical stretching of proteins - A theoretical survey of the Protein Data Bank, J. Phys.: Cond. Mat. 19, 283201 (2007).

${ }^{14}$ M. Sikora, J. I. Sułkowska, and M. Cieplak, Mechanical strength of 17134 model proteins and cysteine spliknots. PLoS Comp. Biol. 5, e1000547 (2009).

${ }^{15} \mathrm{~B}$. Różycki, Ł. Mioduszewski, and M. Cieplak, Unbinding and unfolding of adhesion protein complexes through stretching: Interplay between shear and tensile mechanical clamps, Proteins: Struct. Funct. Bioinf. 82, 3144-3153 (2014).

${ }^{16}$ A. Galera-Prat, A. Gomez-Sicilia, A. F. Oberhauser, M. Cieplak, and M. Carrion-Vazquez, Understanding biology by stretching proteins: recent progress, Curr. Op. Struct. Biol. 20, 63-69 (2010)

${ }^{17}$ P. G. Wolynes, Symmetry and the energy landscapes of biomolecules, Proc. Natl. Acad. Sci. USA 93, 14249 (1996).

${ }^{18} \mathrm{~J}$. N. Onuchic, Z. Luthey-Schulten, and P. G. Wolynes, Theory of protein folding: the energy landscape perspective, Ann. Rev. Phys. Chem. 48, 545-600 (1997).

${ }^{19}$ E. Alm and D. Baker, Prediction of protein-folding mechanisms from free energy landscapes derived from the native structures, Proc. Natl. Acad. Sci USA 96, 11305-11310 (1999).

${ }^{20} \mathrm{~S}$. Takada, Go-ing for the prediction of protein folding mechanism, Proc. Natl. Acad. Sci. USA 96, 11698-11700 (1999).

${ }^{21}$ C. Micheletti, J. R. Banavar, A. Maritan, and F. Seno, Protein Structures and Optimal Folding from a Geometrical Variational Principle, Phys. Rev. Lett. 82, 3372 (1999).

${ }^{22}$ D. Baker, A surprising simplicity to protein folding, Nature 405, 39-42 (2000).

${ }^{23}$ R.B. Best, Y.G. Chen, and G. Hummer, Slow protein conformational dynamics from multiple experimental structures: the helix/sheet transition of arc repressor, Structure 13, 1755-1763 (2005).

${ }^{24}$ Y.G. Chen and G. Hummer, Slow conformational dynamics and unfolding of the calmodulin C-terminal domain, J. Am. Chem. Soc. 129, 2414-2415 (2007).

${ }^{25}$ F.Q. Zhu and G. Hummer, Gating Transition of Pentameric Ligand-Gated Ion Channels, Biophys. J. 97, 2456-2463 (2009).

${ }^{26}$ I. Bahar, A. R. Atilgan, and B. Erman, Direct evaluation of thermal fluctuations in proteins using a single-parameter harmonic potential, Folding and Design 2, 173-181 (1997).

${ }^{27}$ R. M. Daniel and D. A. Cowan, Biomolecular stability and life at high temperatures, CMLS Cell. Mol. Life Sci. 57, 250-264 (2000).

${ }^{28}$ G. Feller, Protein stability and enzyme activity at extreme bi- 
ological temperatures, J. Phys.: Condens. Matter 22, 323101 (2010).

${ }^{29}$ T. Collins, C. Gerday, and G. Feller, Xylanases, xylanase families and extremophilic xylanases, FEMS Microbiol. Rev. 29 3-23 (2005).

${ }^{30}$ P. Argos, M. G. Rossmann, U. M. Grau, H. Zuber, G. Frank, and J. D. Tratschin, Thermal stability and protein structure, Biochem. 18, 5698-5703 (1979).

${ }^{31} \mathrm{R}$. Jaenicke and G. Boehm, The stability of proteins in extreme environments, Curr. Op. Struct. Biol. 8, 738-748 (1998).

${ }^{32}$ A. Szilagyi and P. Zavodszky, Structural differences between mesoscopic, moderately thermophilic and extermely thermophilic protein subunits: results of a comprehensive survey, Structure 8, 493-504 (2000).

${ }^{33}$ C. Cambillau and J.-M. Claverie, Structural and genomic correlates of hyperthermostability, J. Biol. Chem. 275, 32383-32386 (2000).

${ }^{34}$ G. Feller and C. Gerday, Psychrophilic enzymes: Hot topics in cold adaptation, Nature Reviews Microbiology 1, 200-208 (2003).

${ }^{35}$ D. C. Demirjian, F. Moris-Varas, and C. S. Cassidy, Enzymes from extremophiles, Curr. Op. Chem. Biol. 5, 144-151 (2001).

${ }^{36} \mathrm{~S}$. Bjelic, B. O. Brandsdal, and J. Aqvist, Cold Adaptation of Enzyme Reaction Rates, Biochemistry 47, 10049-10057 (2008).

${ }^{37}$ P. De Maayer, D. Anderson, C. Cary, and D. A. Cowan, Some like it cold: understanding the survival strategies of psychrophiles, EMBO Reports 15, 508-517 (2014).

${ }^{38}$ R. J. Russell, J. M. Ferguson, D. W. Hough, M. J. Danson, and G. L. Taylor, The crystal structure of citrate synthase from the hyperthermophilic archaeon Pyrococcus furiosus at $1.9 \mathrm{~A}$ resolution, Biochemistry 36, 9983-9994 (1997).

${ }^{39}$ S. Remington, G. Wiegand, and R. Huber, Crystallographic refinement and atomic models of two different forms of citrate synthase at 2.7 and $1.7 \mathrm{~A}$ resolution, J. Mol. Biol. 158, 111-152 (1982).

${ }^{40}$ R. J. Russell, U. Gerike, M. J. Danson, D. W. Hough, and G. L. Taylor, Structural adaptations of the cold-active citrate synthase from an Antarctic bacterium, Structure 6, 351-361 (1998).

${ }^{41}$ D. R. Boutz, D. Cascio, J. Whitelegge, L. J. Perry, and T.O. Yeates, Discovery of a thermophilic protein complex stabilized by topologically interlinked chains, J. Mol. Biol. 368, 1332-1344 (2007).

${ }^{42}$ M. Cieplak and T. X. Hoang, Folding of proteins in Go models with angular interactions, Physica A 330, 195-205 (2003).

${ }^{43} \mathrm{~J}$. Tsai, R. Taylor, C. Chothia, and M. Gerstein M, The packing density in proteins: Standard radii and volumes, J. Mol. Biol. 290, 253-266 (1999).

${ }^{44}$ O. Szklarczyk, K. Staron, and M. Cieplak, Native state dynamics and mechanical properties of human topoisomerase $I$ within a structure-based coarse-grained model, Proteins: Struct. Funct. Bioinf. 77, 420-431 (2009).

${ }^{45} \mathrm{~W}$. Kabsch, A discussion of the solution for the best rotation to relate two sets of vectors, Acta Cryst. 32, 827-828 (1978).

${ }^{46} \mathrm{M}$. Cieplak and M. O. Robbins, Nanoindentation of 35 virus capsids in a molecular model: Relating mechanical properties to structure, PLOS ONE 8, e63640 (2013).

${ }^{47} \mathrm{M}$. Cieplak and T.X. Hoang, Universality classes in folding times of proteins, Biophys. J. 84, 475-488 (2003).

${ }^{48}$ G. S. Bell, R. J. M. Russell, H. Connaris, D. W. Hough, M. J. Danson, and G. L. Taylor. Stepwise adaptations of citrate synthase to survival at life's extremes, European Journal of Biochemistry 269, 6250-6260 (2002).

${ }^{49}$ M. Karpusas, B. Branchaud, and S. J. Remington, Proposed mechanism for the condensation reaction of citrate synthase: 1.9A structure of the ternary complex with oxaloacetate and carboxymethyl coenzyme A, Biochemistry 29, 2213-2219 (1990).

${ }^{50} \mathrm{M}$. Cieplak and J. I. Sułkowska, Thermal unfolding of proteins, J. Chem. Phys. 123194908 (2005).

${ }^{51}$ D.A. Beard, K.C. Vinnakota, and F. Wu, Detailed enzyme kinetics in terms of biochemical species: study of citrate synthase, PLoS ONE 3, e1825 (2008).

${ }^{52} \mathrm{C}$. Pfleger and H. Gohlke, Efficient and robust analysis of biomacromolecular flexibility using ensembles of network topologies based on fuzzy noncovalent constraints, Structure 21, 17251734 (2013).

${ }^{53}$ See Supplementary Material Document No......... for Table S1, Fig. S1 and Fig. S2. For information on Supplementary Material, see http://www.aip.org/pubservs/epaps.html 